\title{
PROGNOSE DA DISTRIBUIÇÃO DIAMÉTRICA POR MATRIZ DE TRANSIÇÃO EM FLORESTA APÓS COLHEITA NO LESTE DA AMAZÔNIA
}

\section{PROGNOSIS OF DIAMETRIC DISTRIBUTION BY TRANSITION MATRIX AFTER HARVEST IN A FOREST IN EASTERN AMAZON}

\author{
Leonardo Pequeno Reis ${ }^{1}$ Carlos Moreira Miquelino Eleto Torres ${ }^{2}$ Agostinho Lopes de Souza ${ }^{2}$ \\ Lucas José Mazzei de Freitas ${ }^{3}$ Pamella Carolline Marques dos Reis Reis ${ }^{1}$ Liniker Fernandes da Silva ${ }^{4}$ \\ Rodrigo Vieira Leite ${ }^{5}$
}

\section{RESUMO}

No manejo florestal é importante projetar a distribuição diamétrica ao longo do ciclo de corte para subsidiar as técnicas silviculturais mais adequadas à estrutura da floresta. O objetivo do trabalho foi projetar a distribuição diamétrica ao longo do ciclo de corte utilizando Matriz de Transição Markoviana. Em 1979 foi realizada a colheita seletiva, com intensidade de $72,5 \mathrm{~m}^{3} \mathrm{ha}^{-1}$, em uma área de 64 ha, na Floresta Nacional do Tapajós, Pará. Em 1981 foram instaladas, aleatoriamente, 36 parcelas permanentes de $50 \mathrm{~m}$ x $50 \mathrm{~m}$ medidas sucessivamente em 1982, 1983, 1985, 1987, 1992, 1997, 2007, 2010 e 2012. Para a projeção foram usadas duas matrizes de transição: uma com intervalo de tempo de um ano (1981 a 1982) e outra de dois anos (1981 a 1983). Todas as projeções apresentaram distribuição diamétrica em forma de "J" invertido, com pequena descontinuidade no último ano (2012). Houve aderência da distribuição diamétrica a 5\% de significância entre os dados observados e estimados até aos 11 anos de projeção. A matriz de transição pode ser usada para projetar a distribuição diamétrica após a colheita em pequenos períodos de tempo após a colheita florestal.

Palavras-chave: manejo florestal; modelo de crescimento e produção; cadeia de Markov.

\begin{abstract}
In forest management, it is important to project diametric distribution along the cutting cycle to subsidize the most suitable silvicultural techniques to forest structure. The aim of this work was to project diametric distribution along the cutting cycle using Markov Transition Matrix. In 1979, selective harvest was performed with an intensity of $72.5 \mathrm{~m}^{3} \mathrm{ha}^{-1}$, in a 64 ha area in 'Floresta Nacional do Tapajós' - Pará state (PA). In 1981, 36 permanent plots of 50m x 50m were randomly installed and measured successively in 1982, 1983, 1985, 1987, 1992, 1997, 2007, 2010 and 2012. For the projection, two transition matrices were used: one with time interval of one year (1981 to 1982) and another of two years (1981 to 1983). All projections showed diametric distribution in inverted "J" form, with small discontinuity last year (2012). There was adherence of diametric distribution at 5\% significance between observed and estimated data, up to 11 years of projection. Transition matrix can be used to project diametric distribution after harvest

1 Engenheiro Florestal, Dr., Grupo de Pesquisa em Ecologia Florestal, Instituto de Desenvolvimento Sustentável Mamirauá, Estrada do Bexiga, n. 2.584, Fonte Boa, Cx. Postal 38 69.553-225, Tefé (AM), Brasil. leonardopequenoreis@gmail.com/pamellaca@gmail.com

2 Engenheiro Florestal, Dr., Professor titular do Departamento de Engenharia Florestal, Universidade Federal de Viçosa, Av. Purdue, s/n, Campus Universitário, Edif. Reinaldo de Jesus Araújo, CEP 36570-900, Viçosa (MG), Brasil.carlos.eleto@yahoo.com.br/alsouza@ufv.br

3 Engenheiro Florestal, Dr., Pesquisador da Embrapa Amazônia Oriental, Tv. Doutor Enéas Pinheiro, s/n, Marco, CEP 66095-903, Belém (PA), Brasil. lucas.mazzei@embrapa.br

4 Engenheiro Florestal, Dr., Professor Titular, Universidade Federal do Recôncavo da Bahia, Centro, Rua Rui Barbosa, CEP 44380-000, Cruz das Almas (BA), Brasil. linikerfs@gmail.com

5 Graduando em Engenharia Florestal, Departamento de Engenharia Florestal, Universidade Federal de Viçosa, Av. Purdue, s/n, Campus Universitário, Edif. Reinaldo de Jesus Araújo, CEP 36570-900, Viçosa (MG), Brasil.
\end{abstract}

Recebido para publicação em 17/07/2016 e aceito em 1/03/2017

Ci. Fl., v. 28, n. 2, abr .- jun., 2018 
in short periods of time after the forest harvest.

Keywords: forest management; diametric distribution model; Markov chain.

\section{INTRODUÇÃO}

A modelagem de crescimento e produção florestal abrange desde modelos estatísticos simples de povoamento, pela relação do diâmetro ou altura média com a idade do povoamento, até modelos que utilizam as distâncias entre árvores, índices de sítio e área basal do povoamento. Isso para, empiricamente, simular a competição entre árvores por água, luz e nutrientes (VANCLAY, 1994).

Os modelos de crescimento e produção podem ser classificados como: de povoamento total, de árvores individuais e de distribuição diamétrica, cada um é empregado de acordo com o nível de detalhe desejado. Os modelos de distribuição diamétrica estimam a densidade (árvores ha ${ }^{-1}$ ), por classe de diâmetro, nas idades presentes e futuras. Fundamentam-se em funções de densidade de probabilidade (fdp), como a função Weibull (NASCIMENTO et al., 2015), matrizes de probabilidade de transição (AZEVEDO; SOUZA; JESUS, 1995) e inteligência artificial (DIAMANTOPOULOU et al., 2015). A problemática na construção de modelos em florestas tropicais está nos padrões essencialmente estocásticos da dinâmica florestal que dificulta a modelagem (GLOOR et al., 2009; KLIMAS et al., 2012; GUSTAFSSON et al., 2016).

A análise da distribuição diamétrica ao longo do tempo, pode subsidiar decisões silviculturais como intensidade e ciclo de corte em florestas manejadas, em nível de comunidade e/ou por espécie, permitindo o uso sustentável em diferentes cenários de colheita (REIS et al., 2014). Portanto, modelar a distribuição diamétrica, projetando-a ao longo do ciclo de corte é essencial para o conhecimento mais detalhado da estrutura da floresta e sua produtividade (NORD-LARSEN; CAO, 2006).

ACadeia de Markov ou matriz de transição é uma técnica muito empregada em florestas inequiâneas, usada para descrever os processos de sucessão florestal (PENG; HOU; CHEN, 2010), obtendo resultados que seguem a tendência das distribuições diamétricas típicas das florestas da Amazônia (AZEVEDO et al., 1994; TEIXEIRA et al., 2007; VASCONCELOS; HIGUCHI; DOLIVEIRA, 2009; SOUZA et al., 2014) e em outras regiões do Brasil (AZEVEDO; SOUZA; JESUS, 1995; STEPKA et al., 2010; DALLA LANA et al., 2015), por considerar o ingresso, a mortalidade e a transição inicial de árvores entre as classes diamétricas.

Entretanto, essa técnica não considera as mudanças da mortalidade ao longo do tempo e a passagem de árvores entre as classes fica restrita ao período considerado na construção da matriz (SPATHELF; DURLO, 2001).

Assim, o trabalho objetivou prognosticar a distribuição diamétrica usando matriz de transição Markoviana de uma floresta manejada ao longo de 31 anos no leste da Amazônia para subsidiar decisões silviculturais.

\section{MATERIAL E MÉTODOS}

A área de estudo está localizada na Floresta Nacional do Tapajós, na altura do Km $67\left(55^{\circ} 00^{\prime} \mathrm{W}\right.$, $2^{\circ} 45$ 'S) da Rodovia BR-163, Cuiabá-Santarém. Insere-se no bioma Amazônia e a tipologia é Floresta Ombrófila Densa de terra firme. O clima da região é tropical úmido, com temperatura média anual acima de $26^{\circ} \mathrm{C}$ e é classificado como Ami pelo sistema de Köppen. A umidade relativa média é de $86 \%$ e a precipitação média anual de 1.900 a $2.200 \mathrm{~mm}$. Apresenta topografia plana a ondulada, com ocorrência de solo tipo Latossolo Amarelo Distrófico (COSTA FILHO; COSTA; AGUIAR, 1980; ALVARES et al., 2013).

Na Floresta Nacional do Tapajós, especificamente na área de estudo, Costa Filho, Costa e Aguiar (1980) relataram a colheita seletiva, realizada na década de 1940, de quatro espécies de alto valor comercial: pau-rosa (Aniba roseodora Ducke), maçaranduba (Manilkara huberi (Ducke) A. Chev.), freijó (Cordia goeldiana Huber) e cedro (Cedrela odorata L.).

No ano de 1975, foi realizado um inventário florestal a 100\% de intensidade das espécies madeireiras com dap $\geq 15 \mathrm{~cm}$ em 64 ha e, posteriormente, em 1979, realizada colheita intensiva de 64 espécies madeireiras, com um volume médio de extração de 72,5 $\mathrm{m}^{3} \mathrm{ha}^{-1}$ (COSTA FILHO; COSTA; AGUIAR, 1980).

As espécies que se destacaram em volume colhido à época foram: Hymenaea courbaril L., Cara- 
pa guianensis Aubl., Manilkara huberi, Lecythis lurida (Miers) S. A. Mori., Bertholletia excelsa Humb. \& Bonpl., Astronium lecointei Ducke, Goupia glabra Aubl., Virola michelii Heckel, Erisma uncinatum Warm. e Terminalia amazonia (J. F. Gmel) Exell, que juntas representaram 47,4\% do volume total extraído (COSTA FILHO; COSTA, AGUIAR, 1980; REIS et al., 2010). A colheita foi executada sob dois tratamentos: corte de todas as árvores com dap $\geq 45 \mathrm{~cm}$, em 39 ha; e corte das árvores com dap $\geq 55 \mathrm{~cm}$, em 25 ha (COSTA FILHO; COSTA; AGUIAR, 1980). Entretanto, os tratamentos foram considerados conjuntamente, formando apenas uma comunidade, visto a alta similaridade constatada nas comparações realizadas(REIS et al., 2010)

Em 1981, foram instaladas, aleatoriamente, 36 parcelas permanentes de $50 \mathrm{~m}$ x $50 \mathrm{~m}$ cada uma, em que todas as árvores com dap $\geq 5 \mathrm{~cm}$ foram identificadas botanicamente in loco. Remedições dessas parcelas permanentes ocorreram em 1982, 1983, 1985, 1987, 1992, 1997, 2007, 2010 e 2012.

A amplitude de classe usada foi de $5 \mathrm{~cm}$, obtendo 12 classes de diâmetro. As probabilidades de transição para cada intervalo de medição foram obtidas dividindo-se o número de árvores que morreram, mudaram de classe ou permaneceram na mesma classe pelo número de árvores naquela classe no início do período de crescimento.

A distribuição diamétrica do povoamento, a ser projetada do tempo $t$ para $t+\theta$, e a situação do povoamento no tempo $t+\theta$ podem ser totalmente determinadas pela situação no tempo $t$ e pelo ingresso ocorrido no intervalo de tempo em que se usou o modelo em sua forma matricial (AZEVEDO; SOUZA; JESUS, 1995):

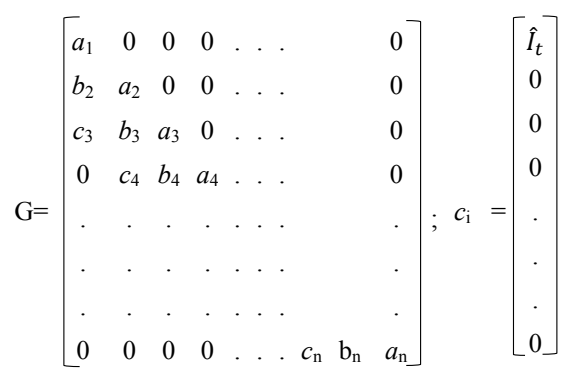

Em que: $\mathrm{a}_{\mathrm{i}}=\mathrm{a}$ probabilidade de uma árvore que está viva numa classe de diâmetro i, no tempo $t$, permaneça viva na mesma classe de diâmetro $i$, no tempo $t+\theta ; b_{i}=$ probabilidade de uma árvore que está viva na classe de diâmetro i- 1 , no tempo t, esteja viva e na classe de diâmetro $i$, no tempo $t+\theta ; c_{i}=$ probabilidade de uma árvore que está viva na classe de diâmetro i-2, no tempo $t$, esteja viva e na classe de diâmetro $i$, no tempo $t+\theta ;$ e $m_{i}=$ probabilidade de uma árvore que está viva na classe $i$, no tempo $t$, esteja morta no intervalo de tempo $t+\theta$; e $\theta=$ período de crescimento entre $t_{0}$ e $t_{1}$.

Onúmero deárvores no final do período de crescimento, em cada classe de diâmetro (BUONGIORNO; MICHIE, 1980; AZEVEDO et al., 1994; AZEVEDO; SOUZA; JESUS, 1995) é dado pela equação matricial de Chapman-Komogorov:

$$
\begin{aligned}
& \mathrm{Y}_{\mathrm{t}+\theta}=\mathrm{G}\left(\mathrm{Y}_{0}\right)+\mathrm{C}_{1}
\end{aligned}
$$

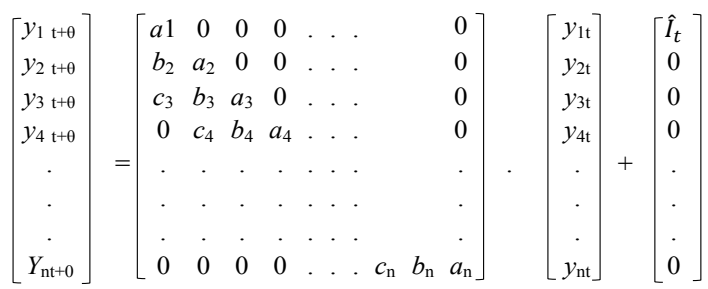

Em que: $\mathrm{Y}_{\mathrm{t}+\theta}=$ vetor coluna do número de árvores por classe de diâmetro, depois de decorrido um período $(\theta) ; \mathrm{G}=$ matriz de probabilidade de transição; $\mathrm{Y}_{\mathrm{it}}=$ vetor coluna número de árvores por classe de diâmetro, no tempo zero; e $\mathrm{C}_{1}=$ vetor coluna de árvores que ingressaram durante um período. 
Para n períodos, pode-se generalizar a equação (2) para:

$$
\mathrm{y}_{\mathrm{n} \theta}=\mathrm{G}^{\mathrm{n}} \cdot \mathrm{y}_{0}+\sum_{\mathrm{i}=0}^{\mathrm{n}-1} \mathrm{G}^{\mathrm{i}} \cdot \mathrm{C}_{(\mathrm{n}-\mathrm{i})}
$$

Os ingressos para cada período de crescimento foram estimados pela equação (AZEVEDO et al., 1994; AZEVEDO; SOUZA; JESUS, 1995):

$$
\hat{\mathrm{I}}_{\mathrm{t}}=\beta_{0}+\beta_{1}\left(\mathrm{~N}_{\mathrm{t}+\theta}-\mathrm{N}_{\mathrm{t}}\right)+\varepsilon_{\mathrm{i}}
$$

Em que: $\hat{I}_{t}=$ é ingresso no período de crescimento; $\mathrm{N}_{\mathrm{t}+\theta}=$ é o número de árvores ha ${ }^{-1}$ no final do período de crescimento; $\mathrm{N}_{\mathrm{t}}=$ é o número de árvores ha ${ }^{-1}$ no início do período de crescimento; e $\varepsilon_{i}=$ erro aleatório. $\beta_{0}$ e $\beta_{1}=$ parâmetros do modelo.

Para a projeção foram considerados dois períodos de tempo, de um ano (1981 a 1982) e de dois anos (1981 a 1983). Foi realizada a projeção para o primeiro período de um ano e comparado com as distribuições diamétricas de 1982, 1983, 1985, 1987, 1992, 1997, 2007 e 2012. Para o período de dois anos foram comparados com as distribuições de 1983, 1985, 1987, 1997 e 2007.

A significância estatística entre as distribuições diamétricas projetada e observada foi verificada pelo teste de aderência Komogorov-Smirnov (K-S), a 5\% de significância, dada pela seguinte equação:

$$
D_{\text {cal. }}=\left|\frac{\operatorname{supF}_{\mathrm{o}} \mathrm{x}-\mathrm{F}_{\mathrm{e}} \mathrm{x}}{\mathrm{n}}\right|
$$

Em que: $\mathrm{F}_{\mathrm{o}}(\mathrm{x})=$ frequência acumulada observada; $\mathrm{F}_{\mathrm{e}}(\mathrm{x})=$ frequência acumulada projetada; $\mathrm{e}$ = número de observações.

$\mathrm{O}$ valor de $\mathrm{D}_{\text {tab. }}$ para $5 \%$ de probabilidade foi obtido pela equação:

$$
\mathrm{D}_{\mathrm{tab}}=\frac{1,35}{\sqrt{\mathrm{n}}}
$$

Em que: $\mathrm{D}_{\text {tab. }}=$ valor crítico a um nível de $5 \%$ de significância. $\mathrm{n}=$ número de observações.

Se $\mathrm{D}_{\text {calc. }}$ for $<\mathrm{D}_{\text {tab. }}=$ aceita-se $\mathrm{H}_{0}$ (distribuição observada é igual à projetada).

Se $\mathrm{D}_{\text {calc. }}$ for $\geq \mathrm{D}_{\text {tab. }}=$ rejeita-se $\mathrm{H}_{0}$ (distribuição observada não é igual à projetada).

Foram avaliados os erros percentuais (Erro \%) em relação aos valores observados por classe de diâmetro:

$$
\text { Erro\% }=\frac{(\widehat{Y}-\mathrm{Y})}{\mathrm{Y}} \cdot 100
$$

Em que: $\hat{Y}=$ valores projetados pela Matriz de Transição na classe de diâmetro; $\mathrm{Y}=$ valores observados nas classes de diâmetro.

\section{RESULTADOS E DISCUSSÃO}

A equação utilizada para estimar o ingresso $\left(\hat{I}_{t}=122,93830+0,73245\left(\mathrm{~N}_{t+\theta}-\mathrm{N}_{t}\right)\right.$ apresentou o $\mathrm{R}^{2}$ de 0,46 e $p$-valor $<0,01$ para a variável independente. Isso ocorre por causa do ingresso ser de difícil modelagem sendo influenciado pelo tamanho das parcelas, intervalo de tempo entre os inventários, tamanho mínimo de inclusão, variabilidade de regeneração, da grande diversidade de espécies e a intensidade da intervenção (ROSSI et al., 2007), mas desconsiderar o recrutamento pode resultar em previsão tendenciosa de crescimento da floresta e seu rendimento futuro (ZHANG et al., 2012).

A matriz de transição G construída (Tabela 1), com um período de tempo de um ano (1981 a 1982) apresenta na diagonal principal a proporção de árvores que permanecerem no mesmo estado, como exemplo, a classe 17,5 e 22,5 cm, respectivamente, proporções de 0,880 e 0,886. A subdiagonal representa a proporção das árvores que se movem para o próximo estado, como exemplo, a classe 17,5 e $22,5 \mathrm{~cm}$, respectivamente, proporções de 0,099 e 0,094. Abaixo da subdiagonal estão as proporções das árvores 
que se movem por mais de um estado, como exemplo, a classe $7,5 \mathrm{~cm}$, com proporção de 0,001 . I é a probabilidade de ocorrência de ingresso na primeira e segunda classe; e M é a probabilidade de mortalidade por classe de diâmetro.

TABELA 1: Matriz de probabilidade de transição, no período de 1981 e 1982. I: probabilidade de ingresso; M: probabilidade de mortalidade em floresta colhida há mais de 30 anos no leste da Amazônia.

TABLE 1: Transition probability matrix, in 1981 and 1982. I: probability of entry; M: probability of mortality in forest harvested in the eastern Amazon.

\begin{tabular}{|c|c|c|c|c|c|c|c|c|c|c|c|c|c|c|}
\hline \multirow{17}{*}{ 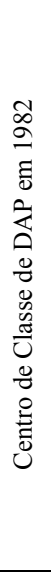 } & & & \multicolumn{12}{|c|}{ Centro de Classe de DAP em 1981} \\
\hline & & I & 7,5 & 12,5 & 17,5 & 22,5 & 27,5 & 32,5 & 37,5 & 42,5 & 47,5 & 52,5 & 57,5 & $\geq 62,5$ \\
\hline & I & & & & & & & & & & & & & \\
\hline & 7,5 & 0,994 & 0,902 & & & & & & & & & & & \\
\hline & 12,5 & 0,006 & 0,071 & 0,890 & & & & & & & & & & \\
\hline & 17,5 & & 0,001 & 0,086 & 0,880 & & & & & & & & & \\
\hline & 22,5 & & & & 0,099 & 0,886 & & & & & & & & \\
\hline & 27,5 & & & & & 0,094 & 0,868 & & & & & & & \\
\hline & 32,5 & & & & & & 0,118 & 0,798 & & & & & & \\
\hline & 37,5 & & & & & & & 0,173 & 0,822 & & & & & \\
\hline & 42,5 & & & & & & & & 0,151 & 0,853 & & & & \\
\hline & 47,5 & & & & & & & & & 0,127 & 0,865 & & & \\
\hline & 52,5 & & & & & & & & & & 0,135 & 0,939 & & \\
\hline & 57,5 & & & & & & & & & & & 0,061 & 1,000 & \\
\hline & $\geq 62,5$ & & & & & & & & & & & & 0 & 0,9818 \\
\hline & $\begin{array}{c}\text { M } \\
\text { Total }\end{array}$ & & 0,026 & 0,023 & 0,022 & 0,020 & 0,014 & 0,029 & 0,027 & 0,020 & 0,000 & 0,000 & 0,000 & 0,018 \\
\hline & $(\mathrm{t})$ & 1 & 1,000 & 1,000 & 1,000 & 1,000 & 1,000 & 1,000 & 1,000 & 1,000 & 1,000 & 1,000 & 1,000 & 1,000 \\
\hline
\end{tabular}

A matriz de transição $G$ apresentou estado inicial absorvente na classe $57,5 \mathrm{~cm}$, caracterizado por não movimento de estado, entrando nesse estado o processo não é alterado ao longo da projeção (SOLOW; SMITH, 2006; BOYCHUK et al., 2009). Outros autores também apresentaram matrizes com estados absorventes como Azevedo et al. (1994), Azevedo, Souza e Jesus (1995), e Stepka et al. (2010). Uma desvantagem da matriz de transição com estado absorvente é por não poder fazer previsões por um período longo e determinar um equilíbrio da distribuição diamétrica (AZEVEDO; SOUZA; JESUS, 1995).

A projeção para n etapas utilizando a matriz de transição construída de 1981 a 1982 (um ano) apresentou até 1992 a aderência com a distribuição diamétrica observada a 5\% de probabilidade (Tabela 2). Já para os anos de 1997, 2007 e 2012, não apresentaram aderência à distribuição diamétrica observada. Como não considera as mudanças da mortalidade ao longo do tempo, a matriz fica limitada a pequenos períodos de projeção.

TABELA 2: Distribuição diamétrica observada (Obs.) e estimada (Est.) com cadeia de Markov, proporção de árvores por classe, usando um período de um ano em floresta colhida há mais de 30 anos no leste da Amazônia. CC.: Centro de classe de diâmetro $(\mathrm{cm})$; Dtab.: valor crítico a 5\% de probabilidade. DCal.: Valor crítico calculado.

TABLE 2: Observed (Obs.) and estimated (Est.) diametric distribution with Markov chain, proportion of trees per class, using a period of one year in forest harvested more than 30 years ago in eastern Amazon. CC.: Class center of diameter $(\mathrm{cm})$; Dtab.: critical value at a level of 5\% probability. DCal.: Calculated critical value.

\begin{tabular}{|c|c|c|c|c|c|c|c|c|c|c|c|c|c|c|c|c|c|}
\hline \multirow{2}{*}{$\mathrm{CC}$} & \multirow{2}{*}{$\begin{array}{l}1981 \\
\text { Obs. }\end{array}$} & \multicolumn{2}{|c|}{1982} & \multicolumn{2}{|c|}{1983} & \multicolumn{2}{|c|}{1985} & \multicolumn{2}{|c|}{1987} & \multicolumn{2}{|c|}{1992} & \multicolumn{2}{|c|}{1997} & \multicolumn{2}{|c|}{2007} & \multicolumn{2}{|c|}{2012} \\
\hline & & Obs. & Est. & Obs. & Est & Obs. & Est. & Obs. & Est. & Obs. & Est. & Obs. & Est. & Obs. & Est. & Obs. & Est. \\
\hline 7,5 & 0,568 & 0,596 & 0,586 & 0,591 & 0,587 & 0,568 & 0,578 & 0,547 & 0,563 & 0,510 & 0,526 & 0,501 & 0,505 & 0,545 & 0,500 & 0,557 & 0,509 \\
\hline 12,5 & 0,175 & 0,168 & 0,173 & 0,179 & 0,176 & 0,188 & 0,177 & 0,191 & 0,175 & 0,195 & 0,156 & 0,187 & 0,127 & 0,175 & 0,071 & 0,172 & 0,050 \\
\hline 17,5 & 0,094 & 0,084 & 0,087 & 0,082 & 0,087 & 0,092 & 0,092 & 0,097 & 0,099 & 0,099 & 0,113 & 0,100 & 0,114 & 0,089 & 0,088 & 0,087 & 0,070 \\
\hline 22,5 & 0,060 & 0,054 & 0,055 & 0,052 & 0,054 & 0,053 & 0,056 & 0,058 & 0,060 & 0,066 & 0,076 & 0,064 & 0,090 & 0,054 & 0,095 & 0,052 & 0,086 \\
\hline 27,5 & 0,035 & 0,032 & 0,032 & 0,031 & 0,031 & 0,032 & 0,031 & 0,037 & 0,033 & 0,044 & 0,042 & 0,047 & 0,053 & 0,040 & 0,068 & 0,038 & 0,069 \\
\hline 32,5 & 0,025 & 0,021 & 0,021 & 0,021 & 0,020 & 0,022 & 0,018 & 0,023 & 0,018 & 0,029 & 0,021 & 0,036 & 0,026 & 0,027 & 0,038 & 0,027 & 0,041 \\
\hline 37,5 & 0,017 & 0,016 & 0,017 & 0,016 & 0,016 & 0,015 & 0,016 & 0,015 & 0,016 & 0,018 & 0,018 & 0,020 & 0,022 & 0,021 & 0,033 & 0,018 & 0,037 \\
\hline 42,5 & 0,012 & 0,012 & 0,012 & 0,011 & 0,012 & 0,010 & 0,012 & 0,012 & 0,013 & 0,012 & 0,016 & 0,014 & 0,019 & 0,015 & 0,028 & 0,015 & 0,033 \\
\hline 47,5 & 0,006 & 0,006 & 0,006 & 0,006 & 0,006 & 0,007 & 0,007 & 0,008 & 0,008 & 0,011 & 0,011 & 0,011 & 0,014 & 0,011 & 0,022 & 0,011 & 0,026 \\
\hline 52,5 & 0,004 & 0,004 & 0,004 & 0,004 & 0,004 & 0,004 & 0,005 & 0,004 & 0,007 & 0,006 & 0,011 & 0,008 & 0,016 & 0,007 & 0,029 & 0,007 & 0,037 \\
\hline 57,5 & 0,002 & 0,002 & 0,002 & 0,002 & 0,002 & 0,002 & 0,002 & 0,002 & 0,003 & 0,003 & 0,006 & 0,004 & 0,010 & 0,007 & 0,025 & 0,007 & 0,036 \\
\hline$\geq 62,5$ & 0,002 & 0,006 & 0,006 & 0,004 & 0,005 & 0,005 & 0,005 & 0,006 & 0,005 & 0,007 & 0,004 & 0,008 & 0,004 & 0,010 & 0,004 & 0,010 & 0,004 \\
\hline $\mathrm{D}_{\text {cal. }}$ & & \multicolumn{2}{|c|}{0,010} & \multicolumn{2}{|c|}{0,008} & \multicolumn{2}{|c|}{0,009} & \multicolumn{2}{|c|}{0,015} & \multicolumn{2}{|c|}{0,022} & \multicolumn{2}{|c|}{0,056} & \multicolumn{2}{|c|}{0,150} & \multicolumn{2}{|c|}{0,185} \\
\hline $\mathrm{D}_{\text {tab. }}$ & & \multicolumn{2}{|c|}{0,041} & \multicolumn{2}{|c|}{0,040} & \multicolumn{2}{|c|}{0,039} & \multicolumn{2}{|c|}{0,039} & \multicolumn{2}{|c|}{0,042} & \multicolumn{2}{|c|}{0,043} & \multicolumn{2}{|c|}{0,041} & \multicolumn{2}{|c|}{0,040} \\
\hline
\end{tabular}


O maior erro percentual foi na classe 17,5 cm em 1982 de 3,8\%, em 1992 e 2012 foi na classe de $57,5 \mathrm{~cm}$, respectivamente, o erro de 90,7 e $433,6 \%$, tendendo a superestimar as frequências nas maiores classes, principalmente na classe absorvente. Isso provoca um aumento gradual e crescente no número de árvores na projeção para as maiores classes de diâmetro, devido ao crescimento contínuo empregado pela matriz de transição nessas classes (SANQUETTA et al., 1997).

Essa característica tem relevância na aplicação do manejo florestal, por apresentar contínuo crescimento do volume e área basal, causada pelo aumento de árvores nas maiores classes. Superestimando o estoque a ser colhido na floresta, limitando o uso da matriz como ferramenta para o manejo ao longo prazo.

Quanto mais etapas ao longo do tempo, maior o erro de projeção da matriz, principalmente pelo longo processo de regeneração da floresta colhida e pelas mudanças que ocorrem na estrutura diamétrica, como a mudança na composição florística e no crescimento, como apresentado por Reis et al. (2010). Isso indica que as aplicações de cortes, por si só, conduzem o povoamento para alguma situação diferente da situação inicial (AZEVEDO et al., 1994). De forma análoga, a dinâmica da sucessão natural provavelmente não restitui igual situação à apresentada pela matriz.

A estrutura diamétrica em forma de "J" invertido se mantém ao longo da projeção (Figura 1), com variação a partir de 2007, principalmente nas classes de 12,5 e 17,5 cm, com diminuição do percentual de número de árvores nessas classes, isso corrobora os resultados obtidos por Azevedo et al. (1994) em floresta manejada.
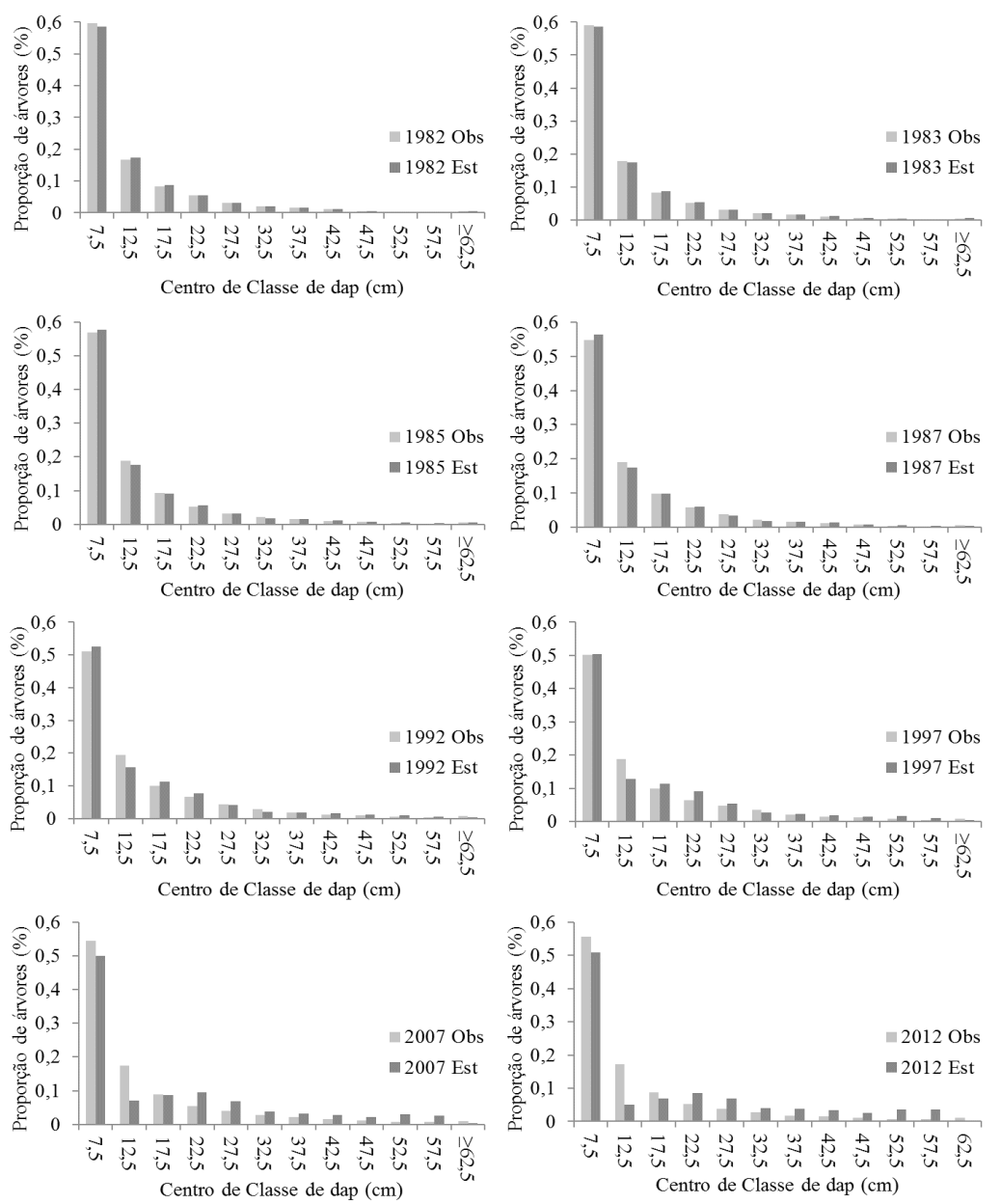

FIGURA 1: Distribuição diamétrica, proporção de árvores por classe, observadas (Obs.) e estimadas (Est.) com a cadeia de Markov (um ano), em uma floresta colhida há mais de 30 anos no leste da Amazônia.

FIGURE 1: Diametric distribution, proportion of trees per class, observed (Obs.) and estimated (Est.) with the Markov chain (one year), in a forest harvested more than 30 years ago in eastern Amazon. 
A tendência "J" invertido somente foi mantida por causa do ingresso estimado pela equação, sem o ingresso essa estrutura seria mais uniforme até a classe absorvente, com aumento no número de árvores nessa classe. Sem essa classe absorvente, a projeção seguiria para um estado estável, dessa forma a proporção de árvores por classe de diâmetro será a mesma (BUONGIORNO; GILLESS, 1987).

Para o período de transição de dois anos (1981 a 1983), a matriz de transição não apresentou um estado absorvente (Tabela 3), sendo um ganho em comparação com a matriz de um ano. Isso ocorre por representar melhor a dinâmica da floresta, contendo a passagem de classe por dois estados, como por exemplo, as classes de 7,5, 12,5 e 32,5 cm; e também a mortalidade melhor incorporada na matriz nesse intervalo de tempo. Azevedo, Souza e Jesus (1995) observaram que em intervalos de tempo ( $\theta$ ) maiores na construção das matrizes podem-se obter melhores estimativas.

TABELA 3: Matriz de probabilidade de transição, no período de 1981 e 1983. I: probabilidade de ingresso; M: probabilidade de mortalidade em floresta colhida há mais de 30 anos no leste da Amazônia.

TABLE 3: Transition probability matrix, in 1981 and 1983. I: probability of entry; M: probability of mortality, in forest harvested more than 30 years ago in eastern Amazon.

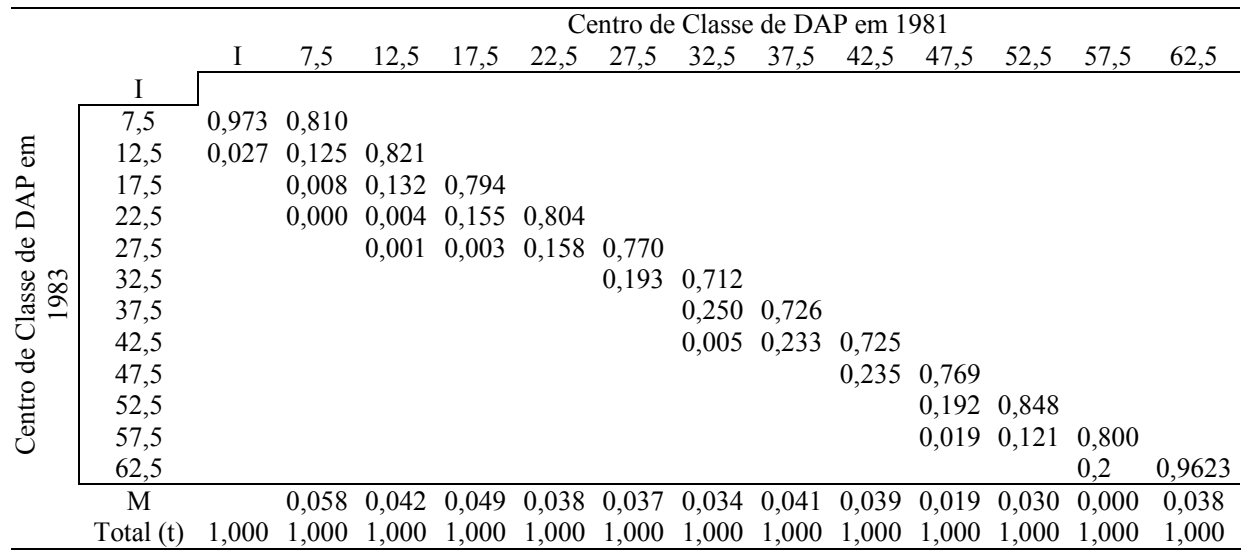

A forma característica de "J" invertido foi observada para todas as projeções, isso por representar melhor a dinâmica nas menores classes, mas com uma descontinuidade no ano 2007 (Figura 2). Os valores do $\mathrm{D}_{\text {cal }}$ do teste K-S, para os anos de 1997 e 2007 foram menores do que a matriz de passagem de um ano (1981 a 1982) no mesmo período, isso significa que a matriz de dois anos apresenta menor diferença na prognose da distribuição diamétrica.

Os maiores erros percentuais para a projeção da distribuição nos diferentes anos foram: no ano de 1983 de 10,2\% (17,5 cm), em 1985 de 15,5\% (42,5 cm), em 1987 de 19,2\% (52,5 cm), em 1997 de 34,2\% $(22,5 \mathrm{~cm})$, e em 2007 de $127,2 \%(52,5 \mathrm{~cm})$, também tende a superestimar a frequência nas maiores classes.

Os resultados do teste K-S mostraram que para ambas as projeções com matriz de intervalo de um ano e de dois anos, as maiores diferenças ocorreram nas menores classes de diâmetro, por serem as classes mais dinâmicas em crescimento e mortalidade. Resultado semelhante também foi observado em Floresta Atlântica não manejada por Dalla Lana et al. (2015). Quanto mais dinâmica a floresta, mais a matriz fica limitada por representar somente o início da dinâmica após a colheita.

A projeção da distribuição diamétrica usando o tempo de dois anos, apresentou aderência a 5\% de significância até o ano de 1987 (Tabela 4). Para os anos de 1997 e 2007, a distribuição projetada se diferenciou da observada, seguindo o mesmo padrão da matriz de um ano. Demonstrando, mesmo com o intervalo maior, a limitação da matriz de probabilidade de transição em simular a dinâmica florestal após a colheita de alta intensidade para os parâmetros atuais. A colheita de alta intensidade aumenta a dinâmica entre as classes no primeiro momento e diminui gradualmente com o passar do tempo com o fechamento do dossel florestal. 
TABELA 4: Distribuição diamétrica observada (Obs.) e estimada (Est.) com cadeia de Markov, proporção de árvores por classe, usando um período de dois anos, em floresta colhida há mais de 30 anos no leste da Amazônia. CC.: Centro de classe de diâmetro (cm); Dtab.: valor crítico a de 5\% de probabilidade. DCal.: Valor crítico calculado.

TABLE 4: Observed (Obs.) and estimated (Est.) diametric distribution with Markov chain, proportion of trees per class, using a period of two years in forest harvested more than 30 years ago in eastern Amazon. CC.: Class center of diameter (cm); Dtab.: critical value at a level of $5 \%$ probability. DCal.: Calculated critical value.

\begin{tabular}{|c|c|c|c|c|c|c|c|c|c|c|c|}
\hline \multirow{2}{*}{$\mathrm{CC}$} & 1981 & \multicolumn{2}{|c|}{1983} & \multicolumn{2}{|c|}{1985} & \multicolumn{2}{|c|}{1987} & \multicolumn{2}{|c|}{1997} & \multicolumn{2}{|c|}{2007} \\
\hline & Obs. & Obs. & Est. & Obs. & Est. & Obs. & Est. & Obs. & Est. & Obs. & Est. \\
\hline 7,5 & 0,568 & 0,591 & 0,556 & 0,568 & 0,535 & 0,547 & 0,518 & 0,501 & 0,482 & 0,545 & 0,504 \\
\hline 12,5 & 0,175 & 0,179 & 0,195 & 0,188 & 0,206 & 0,191 & 0,208 & 0,187 & 0,161 & 0,175 & 0,089 \\
\hline 17,5 & 0,094 & 0,082 & 0,091 & 0,092 & 0,097 & 0,097 & 0,104 & 0,100 & 0,120 & 0,089 & 0,098 \\
\hline 22,5 & 0,060 & 0,052 & 0,057 & 0,053 & 0,058 & 0,058 & 0,062 & 0,064 & 0,087 & 0,054 & 0,095 \\
\hline 27,5 & 0,035 & 0,031 & 0,033 & 0,032 & 0,034 & 0,037 & 0,035 & 0,047 & 0,049 & 0,040 & 0,065 \\
\hline 32,5 & 0,025 & 0,021 & 0,022 & 0,022 & 0,021 & 0,023 & 0,021 & 0,036 & 0,027 & 0,027 & 0,039 \\
\hline 37,5 & 0,017 & 0,016 & 0,017 & 0,015 & 0,017 & 0,015 & 0,017 & 0,020 & 0,021 & 0,021 & 0,030 \\
\hline 42,5 & 0,012 & 0,011 & 0,012 & 0,010 & 0,012 & 0,012 & 0,013 & 0,014 & 0,016 & 0,015 & 0,022 \\
\hline 47,5 & 0,006 & 0,006 & 0,007 & 0,007 & 0,008 & 0,008 & 0,009 & 0,011 & 0,013 & 0,011 & 0,019 \\
\hline 52,5 & 0,004 & 0,004 & 0,004 & 0,004 & 0,005 & 0,004 & 0,005 & 0,008 & 0,010 & 0,007 & 0,017 \\
\hline 57,5 & 0,002 & 0,002 & 0,002 & 0,002 & 0,002 & 0,002 & 0,002 & 0,004 & 0,005 & 0,007 & 0,008 \\
\hline$\geq 62,5$ & 0,002 & 0,005 & 0,006 & 0,005 & 0,006 & 0,006 & 0,006 & 0,008 & 0,008 & 0,010 & 0,013 \\
\hline $\mathrm{D}_{\text {cal. }}$ & & \multicolumn{2}{|c|}{0,035} & \multicolumn{2}{|c|}{0,034} & \multicolumn{2}{|c|}{0,029} & \multicolumn{2}{|c|}{0,045} & \multicolumn{2}{|c|}{0,127} \\
\hline $\mathrm{D}_{\text {tab. }}$ & & \multicolumn{2}{|c|}{0,041} & \multicolumn{2}{|c|}{0,040} & \multicolumn{2}{|c|}{0,039} & \multicolumn{2}{|c|}{0,039} & \multicolumn{2}{|c|}{0,042} \\
\hline
\end{tabular}
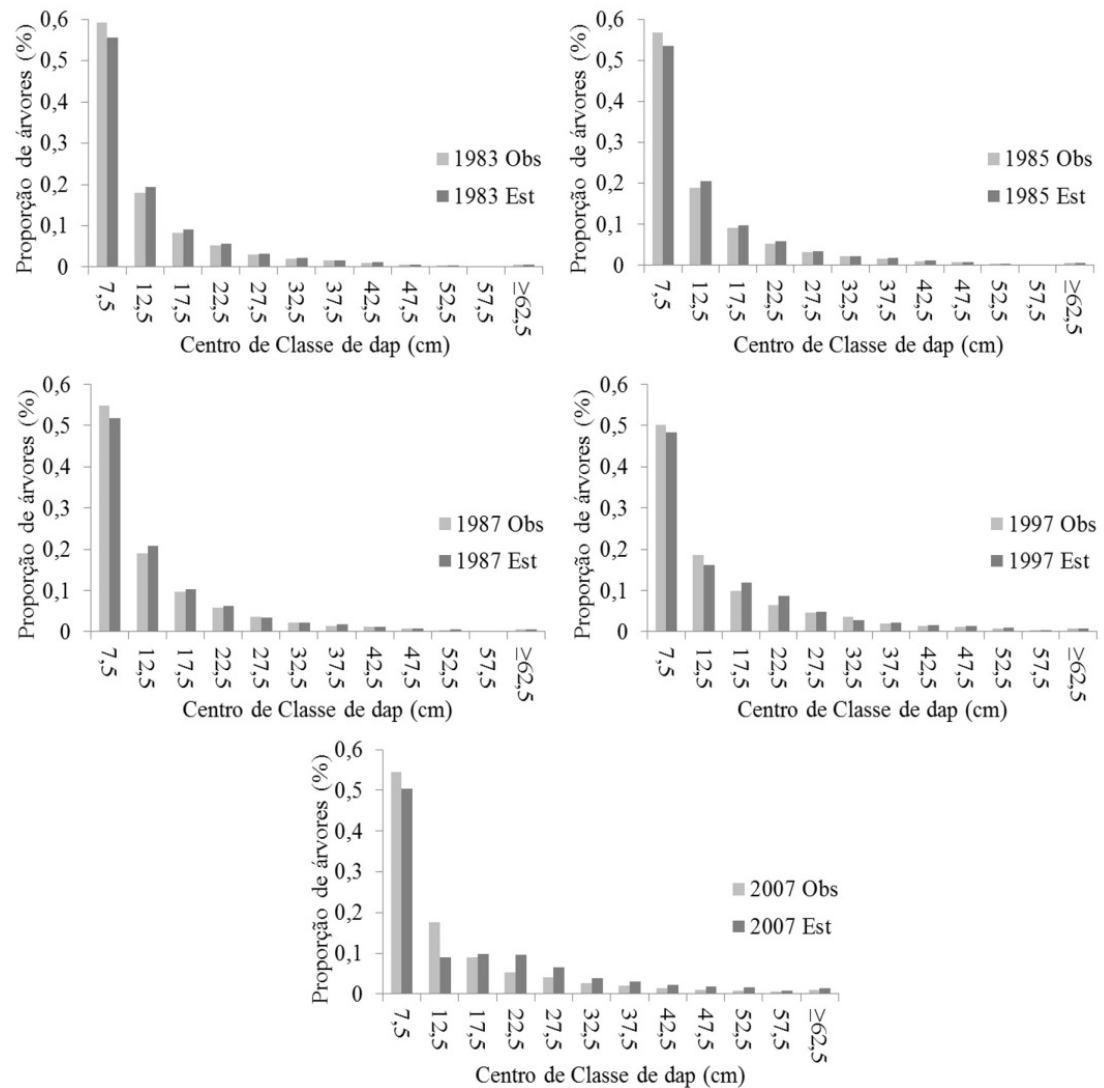

FIGURA 2: Distribuição diamétrica, proporção de árvores por classe, observadas (Obs.) e estimadas (Est.) com a cadeia de Markov (dois anos), em uma floresta colhida há mais de 30 anos no leste da Amazônia.

FIGURE 2: Diametric distribution, proportion of trees per class, observed (Obs.) and estimated (Est.) with Markov chain (two years), in a forest harvested more than 30 years ago in eastern Amazon. 
Vale ressaltar que para tratamentos silviculturais, as matrizes apresentadas servem como ferramenta de decisão, pelo menos, para períodos de tempo curto, se a floresta apresentar taxas de crescimento lento (SOARES et al., 2009). Servindo como um guia inicial de apoio a decisões de intensidade de corte e de uso de técnicas silviculturais, como, por exemplo, de refinamento, que pode utilizar a distribuição diamétrica para reduzir, por classe de diâmetro, a área basal de forma sistemática, como ocorre na técnica BDq (WEAVER et al., 2009; HANSON et al., 2012).

Também é possível a cada período, utilizando-se de inventário em parcelas permanentes, caso seja objetivo do manejador, atualizar a matriz de transição e ir projetando em pequenos períodos, dessa forma a incerteza em relação à estrutura será reduzida.

\section{CONCLUSÕES}

A matriz de transição representa a dinâmica da distribuição diamétrica até 11 anos após a colheita, servindo como um guia inicial para recomendação de intensidade de corte e tratamentos silviculturais.

A utilização da matriz após a colheita não deve ser usada em períodos maiores de 15 anos, por apresentar diferenças na estrutura em forma de "J" invertido.

\section{AGRADECIMENTOS}

A Capes e ao CNPq pela concessão de bolsas aos autores e a Embrapa Amazônia Oriental.

\section{REFERÊNCIAS}

ALVARES, C. A. et al. Koppen's climate classification map for Brazil. Meteorologische Zeitschrift, Stuttgart, v. 22, p. 711-728, 2013. doi:10.1127/0941-2948/2013/0507

AZEVEDO, C. P. et al. Predição da distribuição diamétrica de povoamento florestais inequiâneo pelo emprego da matriz de transição. Revista Árvore, Viçosa, MG, v. 18, n. 3, p. 179-193, 1994.

AZEVEDO, C. P.; SOUZA, A. L.; JESUS, R. M. Um modelo de matriz de transição para prognose de crescimento de povoamento natural remanescente de Mata Atlântica. Revista Árvore, Viçosa, MG, v. 19, n. 2, p. 167-199, 1995.

BOYCHUK, D. et al. A stochastic forest fire growth model. Environmental and Ecological Statistics, University Park, v. 16, n. 2, p. 133-151, 2009. 10.1007/s10651-007-0079-z

BUONGIORNO, J.; GILLES, J. K. Forest management and economics: a primer in quantitative methods. New York: Macmillan, 1987. 285 p.

BUONGIORNO, J.; MICHIE, B. R. A matrix model of uneven-aged forest management. Forest Science, Madison, v. 26, n. 4, p. 609-625, 1980.

COSTA FILHO, P. P.; COSTA, H. B.; AGUIAR, O. R. Exploração mecanizada da floresta tropical, úmida sem babaçu. Belém: Embrapa Amazônia Oriental, 1980. 29 p. (Circular técnico, 9).

DALLA LANA, M. et al. Prognose da estrutura diamétrica em Floresta Ombrófila Mista. Floram, Seropédica, v. 22, n. 1, p. 71-78, 2015. 0.1590/2179-8087.072914

DIAMANTOPOULOU, M. J. et al. Estimation of Weibull function parameters for modelling tree diameter distribution using least squares and artificial neural networks methods. Biosystems Engineering, Bedford, v. 133, p. 33-45, 2015. 10.1016/j.biosystemseng.2015.02.013

GLOOR, M. et al. Does the disturbance hypothesis explain the biomass increase in basin-wide Amazon forest plot data? Global Change Biology, Malden, v. 15, p. 2418-2430, 2009. 10.1111/j.1365-2486.2009.01891.x GUSTAFSSON, M. et al. Life history traits predict the response to increased light among 33 tropical rainforest tree species. Forest Ecology and Management, Amsterdam, v. 362, p. 20-28, 2016. 10.1016/j. dib.2015.12.048

HANSON, J. J. et al. Ecological forestry in an uneven-aged, late-successional forest: simulated effects of contrasting treatments on structure and yield. Forest Ecology and Management, Amsterdam, v. 270, p. 94-107, 2012. 10.1016/j.foreco.2012.01.017

KLIMAS, C. A. et al. Viability of combined timber and non-timber harvests for one species: A Carapa 
guianensis case study. Ecological Modelling, Amsterdam, v. 246, p. 147-156, 2012. 10.1016/j. ecolmodel.2012.07.022

NASCIMENTO, R. G. M. et al. A growth and yield projection system for a tropical rainforest in the Central Amazon, Brazil. Forest Ecology and Management, Amsterdam, v. 327, p. 201-208, 2015. DOI: 10.1016/j. foreco.2014.05.007

NORD-LARSEN, T.; CAO, Q. V. A diameter distribution model for even-aged beech in Denmark. Forest Ecology and Management, Amsterdam, v. 231, n. 1/3, 2006. 10.1016/j.foreco.2006.05.054

PENG, S. L.; HOU, Y. P.; CHEN, B. M. Establishment of Markov successional model and its application for forest restoration reference in Southern China. Ecological Modelling, Amsterdam, v. 221, n. 9, p. 13171324, 2010. 10.1016/j.ecolmodel.2010.01.016

REIS, L. P. et al. Avaliação do potencial madeireiro na Floresta Nacional do Tapajós após 28 anos da exploração florestal. Pesquisa Florestal Brasileira, Colombo, v. 30, n. 64, p. 265-281, 2010. 10.4336/2010. pfb.30.64.265

REIS, L. P. et al. Dinâmica da distribuição diamétrica de algumas espécies de Sapotaceae após exploração florestal na Amazônia Oriental. Revista de Ciências Agrárias, Belém, v. 57, p. 234-243, 2014. 10.4322/ rca.ao1401

ROSSI, L. M. B. et al. Modelagem de Recrutamento em Florestas. Floresta, Curitiba, v. 37, p. 453-467, 2007. 10.5380/rf.v37i3.9942

SANQUETTA, C. R. et al. Análise de sensibilidade e de risco em prognose volumétrica de florestas com matriz de transição. Revista do setor de ciências agrárias, Curitiba, v. 16, n. 1/2, p. 47-62, 1997.

SOARES, C. P. B. et al. Predição do crescimento em povoamentos clonais de eucalipto não desbastados utilizando matrizes de transição não estacionárias. Revista Árvore, Viçosa, MG, v. 33, n. 5, p. 831-840, 2009. 10.1590/S0100-67622009000500006

SOLOW, R. S.; SMITH, W. K. Using Markov chain successional models backwards. Journal of Applied Ecology, London, v. 43, p. 185-188, 2006.

SOUZA, C. R. et al. Projection of diametric distribution and carbon stock of a managed forest in Manaus/ AM. Floresta, Curitiba, v. 44, p. 525-534, 2014. 10.5380/rf.v44i3.32854

SPATHELF, P.; DURLO, M. A. Transition matrix for modeling the dynamics of a subtropical seminatural forest in southern Brazil. Forest Ecology and Management, Amsterdam, v. 151, n. 1-3, p. 139-149, 2001. 10.1016/S0378-1127(00)00704-0

STEPKA, T. F. et al. Prognose da estrutura diamétrica de uma Floresta Ombrófila Mista com os métodos Razão de Movimentos e Matriz de Transição. Pesquisa Florestal Brasileira, Colombo, v. 30, n. 64, p. 327336, 2010. 10.4336/2010.pfb.30.64.327

TEIXEIRA, L. M. et al. Projeção da dinâmica da floresta natural de terra firme, região de Manaus-AM, com o uso da Cadeia de Transição Probabilística de Markov. Acta Amazonica, Manuas, v. 37, n. 3, p. 377-384, 2007. 10.1590/S0044-59672007000300009

VANCLAY, J. K. Modelling forest growth and yield: applications to mixed tropical forests. Oxford: CAB International, 1994. $312 \mathrm{p}$.

VASCONCELOS, S. S.; HIGUCHI, N.; DOLIVEIRA, M. V. N. Projeção da distribuição diamétrica de uma floresta explorada seletivamente na Amazônia Ocidental. Acta Amazonica, Manaus, v. 31, n. 1, p. 71-80, 2009. DOI: 10.1590/S0044-59672009000100007

WEAVER, J. K. et al. Decaying wood and tree regeneration in the Acadian Forest of Maine, USA. Forest Ecology and Management, Amsterdam, v. 257, n. 7, p. 1623-1628, 2009. 10.1016/j.foreco.2009.01.023 ZHANG, X. et al. Predicting tree recruitment with negative binomial mixture models. Forest Ecology and Management, Amsterdam, v. 270, p. 209-215, 2012. 10.1016/j.foreco. 2012.01.028. 